\title{
Elliptic Curves Over Finite Fields. II
}

\author{
By I. Borosh, C. J. Moreno and H. Porta
}

\begin{abstract}
The class groups of certain elliptic function fields without complex multiplications are computed. Questions about the structure of these groups and the arithmetical nature of their orders are considered.
\end{abstract}

1. Introduction. The present work gives in greater detail the computations outlined in the Boulder paper [2]. Let $E$ be an elliptic curve whose Néron minimal model is

$$
E: y^{2}+a_{1} x y+a_{3} y=x^{3}+a_{2} x^{2}+a_{4} x+a_{6}
$$

with $a_{i} \in \mathbf{Z}$ and of conductor $N$. For a fixed prime $p$, the same equation for $E$ with coefficients read in the finite field $\mathbf{F}_{p}$ of $p$ elements defines an elliptic curve $E\left(\mathbf{F}_{p}\right)$ over the algebraic closure $\overline{\mathbf{F}}_{p}$ of $\mathbf{F}_{p}$ for all primes $p$ not dividing the conductor $N$. In this paper we study that part of $E\left(\overline{\mathbf{F}}_{p}\right)$ which is left fixed by the action of the Galois group $G=\operatorname{Gal}\left(\overline{\mathbf{F}}_{p} / \mathbf{F}_{p}\right)$. More precisely, we study the structure of the finite abelian group,

$$
E\left(\mathrm{~F}_{p}\right): y^{2}+a_{1} x y+a_{3} y \equiv x^{3}+a_{2} x^{2}+a_{4} x+a_{6} \bmod (p),
$$

consisting of the points on the elliptic curve $E$ whose coordinates lie in the finite field $\mathbf{F}_{p}$ and also the point at infinity. We will not consider the somewhat simpler question of the structure of $E\left(\mathrm{~F}_{p}\right)$ for those primes $p$ which divide the conductor of $E$, since this can be done mechanically once the Kodaira type of the reduced fiber is known.

The starting point of our investigations was the important work of Shimura [11] where knowledge of the number of points on the curve,

$$
E\left(\mathrm{~F}_{p}\right): y^{2} \equiv 4 x^{3}-\left(\frac{4 \cdot 31}{3}\right) x-\left(\frac{41 \cdot 61}{27}\right) \bmod (p),
$$

was used to obtain information about the nonsolvable field extensions obtained by adjoining to the rationals the coordinates of the $l$-division points on the curve,

$$
y^{2}=4 x^{3}-\left(\frac{4 \cdot 31}{3}\right) x-\left(\frac{41 \cdot 61}{27}\right)
$$

which as Tate has observed is isogenous to $y^{2}-y=x^{3}-x^{2}$. The computations given by Shimura in [11] for

$$
N_{p}=\operatorname{Card} E\left(\mathbf{F}_{p}\right)=p-a_{p}+1
$$

Received February 11, 1974; revised April 29, 1974.

AMS (MOS) subject classifications (1970). Primary 14G15; Secondary $10 \mathrm{D} 99$.

Key words and phrases. Elliptic curves, modular forms, finite fields, traces of Frobenius. 
were obtained by computing the coefficients $a_{n}$ in the infinite product,

$$
f(z)=q \prod_{n=1}^{\infty}\left(1-q^{n}\right)^{2}\left(1-q^{11 n}\right)^{2}=\sum_{n=1}^{\infty} a_{n} q^{n}, \quad q=\exp (2 \pi i z),
$$

which is a cusp form of weight 2 associated with the Hecke congruence subgroup $\Gamma_{0}(11)$. The traces of Frobenius $a_{p}$ in Shimura [11] are given for primes $p \leqslant 2000$.

D. H. Lehmer also computed the $a_{p}$ using the above product expansion for all primes $p \leqslant 30000$; incidentally, Lehmer found many primes $p$ for which $a_{p}=0$.

There are in nature eleven other curves like the one considered by Shimura which are uniformized by modular forms on $\Gamma_{0}(N)$. Affine models for these have been given by Birch [1]. Thus in principle one can compute Card $E\left(\mathbf{F}_{p}\right)$ in essentially two different ways: namely, by counting the number of points on the Birch models (unfortunately, these are not given in Néron minimal form!) and by computing the traces of Frobenius via an explicit construction of the cusp form associated with the modular curve which can be obtained as a linear combination of suitable theta functions.

For $N=14$ Birch [1] has given the model,

$$
E_{14}: y^{2}+x y=x^{3}+3 x^{2}+8 x .
$$

The associated cusp form of weight 2 and level 14 as given by Doi and Naganuma [7] is

$$
-\sum_{n=1}^{\infty} a_{n} q^{n}=q \prod_{n=1}^{\infty}\left(1-q^{n}\right)\left(1-q^{2 n}\right)\left(1-q^{7 n}\right)\left(1-q^{14 n}\right) .
$$

In [10] Serre has given an affine model of a curve of conductor 14,

$$
E_{s}: y^{2}+x y+y=x^{3}-x .
$$

Our preliminary computations gave that the traces of Frobenius for primes $p \leqslant 5000$ were identical for both curves $E_{14}$ and $E_{s}$. Also, for all primes $p \leqslant 2000$ we computed the groups $E_{14}\left(\mathrm{~F}_{p}\right)$ and $E_{s}\left(\mathrm{~F}_{p}\right)$ and found that they agree except for the splitting of the 3-primary component. This suggested strongly the existence of an isogeny of degree 3. That this is in fact true can be explained by observing that the elliptic curve $E_{s}$ is in fact the curve $X_{1}(14)$ in the notation of Ogg [9], $E_{14}=X_{0}(14)$, and $X_{1}(14)$ covers $X_{0}(14)$ by an isogeny of degree 3. More explicitly, $E_{s}$ is equivalent to $y^{2}+x y$ $-y=x^{3}$; and dividing out by the cyclic group of order 3 generated by $(0,0)$, we get $y^{2}+x y=x^{3}+3 x^{2}+8 x$ which is Birch's equation. We might add that the general rule for dividing $y^{2}+a_{1} x y+a_{3} y=x^{3}$ by the point $(0,0)$ is $y^{2}+a_{1} x y+3 a_{3} y-$ $6 a_{1} a_{3} x+a_{1}^{2} a_{3}-9 a_{3}^{2}$.

The contents of the paper are as follows. In Section 2 we give a brief description of the group law on $E\left(\mathbf{F}_{p}\right)$ which is useful in computation. In Section 3 we describe a method for computing the primary decomposition of $E\left(F_{p}\right)$ by machine. In Section 4 we present the numerical results obtained. In this same section we also give various conjectures and theorems which were suggested by the machine computations. The primary decomposition of $E\left(\mathbf{F}_{p}\right)$ for various curves and primes $p \leqslant 5000$ and also for some other primes $p \leqslant 5000$ are given at the end of the paper.

The present version of the paper owes much to the suggestions of many people. 
Here we would like to record our thanks to the referee, who among other things pointed out the isogeny between $E_{14}$ and $E_{s}$ given above and also suggested the first and third remarks which appear at the end of Section 3.

2. The Group Law. When the elliptic curve $E$ is given in Weierstrass normal form,

$$
E: y^{2}=4 x^{3}-g_{2} x-g_{3},
$$

with $g_{2}, g_{3} \in \mathbf{Z}$, the group law for $E$ in characteristic zero corresponds simply to the addition formulas for the Weierstrass $p$-function,

$$
p(z)=z^{-2}+\sum_{\omega \in \Omega}^{\prime}\left((z-\omega)^{-2}-\omega^{-2}\right) .
$$

Now if the elliptic curve $E$ has good reduction at a prime $p$, the formula for the group law of $E$ can be reduced modulo $p$ to give the group law for $E\left(\mathrm{~F}_{p}\right)$. This procedure works for almost all primes not dividing the conductor. To obtain formulas defining the group law for $E\left(\mathbf{F}_{p}\right)$ which work for all primes not dividing the conductor one must work with the Néron minimal model of $E$ and obtain explicit formulas in characteristic zero and then read them modulo the prime $p$ to obtain the group law for $E\left(\mathrm{~F}_{p}\right)$.

In characteristic zero the group law is obtained by the tangent-chord process of Euler: "Three collinear points on $E$ add up to zero," where the zero element on $E$ is taken to be the point of infinity $P_{\infty}=(\infty, \infty)$. Thus, if $P_{1}=\left(z_{1}, y_{1}\right)$ and $P_{2}=\left(x_{2}, y_{2}\right)$ are two distinct points on $E$, their sum $P_{3}=\left(x_{3}, y_{3}\right)$ is the inverse of the point $-P_{3}=$ ( $x_{3}^{*}, y_{3}^{*}$ ) where the curve $E$ intersects the line passing through the points $P_{1}$ and $P_{2}$. To obtain $P_{3}$ from $-P_{3}$ one considers a line passing through $-P_{3}, P_{3}$, and $P_{\infty}$. For an elliptic curve with affine model,

$$
E: y^{2}+a_{1} x y+a_{3} y=x^{3}+a_{2} x^{2}+a_{4} x+a_{6},
$$

the coordinates of $P_{3}=\left(x_{3}, y_{3}\right)$ are given by

$$
x_{3}=-x_{1}-x_{2}+m^{2}+a_{1} m-a_{2}, \quad y_{3}=-y_{1}-m x_{3}+x_{1} m-a_{1} x_{3}-a_{3},
$$

where $m=\left(y_{2}-y_{1}\right) /\left(x_{2}-x_{1}\right)$. The double of a point, i.e. the case $P_{1}=P_{2}$, is found similarly by first observing that the tangent to $E$ at $P_{1}$ has a contact of second order (the intersection multiplicity is 2 ) and then finding the other point of intersection. The coordinates of $2 P_{1}=P=(x, y)$ are

$$
x=-2 x_{1}+m^{2}+a_{1} m-a_{2}, \quad y=-a_{1} x-a_{3}-y_{1}-m\left(x-x_{1}\right),
$$

where

$$
m=\left(3 x_{1}^{2}+2 a_{2} x_{1}+a_{4}-a_{1} y_{1}\right) /\left(2 y_{1}+a_{1} x_{1}+a_{3}\right) .
$$

The group law for $E\left(\mathrm{~F}_{p}\right)$ is now given by the above formulas read modulo $p$. The good reduction of the elliptic curve $E$ at a prime $p$ which does not divide the conductor of $E$ guarantees that the group law for $E\left(\mathrm{~F}_{p}\right)$ given by the above formulas are well defined modulo $p$.

The $l$-division equation plays a very important role in the following considerations. If $t=(x(t), y(t))$ is a point on the elliptic curve, then the $l$ th multiple $l t$ of the point $t$ 
has coordinates $l t=(x(l t), y(l t))$, where

$$
x(l t)=B_{l}(x) / A_{l}(x)^{2} \text { and } y(l t)=y D_{l}(x) / A_{l}(x)^{3} .
$$

The polynomial $A_{l}(x)$ is of degree $\left(l^{2}-1\right) / 2$ and is classically known as the $l$-division equation. For small $l$ it can be computed by iterating the group law. For more details see the expository article by Cassels [5].

\section{Machine Computations.}

\subsection{Computation of the Points and the Order of the Group. Let}

$$
E\left(\mathbf{F}_{p}\right): y^{2}+a_{1} x y+a_{3} y=x^{3}+a_{2} x^{2}+a_{4} x+a_{6}
$$

be the reduction mod $p$ of the Néron minimal model for $E$. To compute the points on $E\left(\mathbf{F}_{p}\right)$, we take a $\xi$ in $\mathbf{F}_{p}$ and solve for $y$ the quadratic equation,

$$
y^{2}+\left(a_{1} \xi+a_{3}\right) y+\left(-\xi^{3}-a_{2} \xi^{2}+a_{4} \xi+a_{6}\right)=0,
$$

using the formal expression $y=\left(-a_{1} \xi-a_{3} \pm \Delta^{1 / 2}\right) / 2$, where

$$
\Delta=\left(a_{1} \xi+a_{3}\right)^{2}+4\left(\xi^{3}+a_{2} \xi^{2}+a_{4} \xi+a_{6}\right) \text {. }
$$

This process yields two points on the curve, one point, or no point depending on whether $\Delta$ is a nonzero square in $\mathbf{F}_{p}$, zero or a nonsquare. The repeated use of this algorithm for all $\xi=\mathrm{F}_{p}$ gives all the points on $E\left(\mathrm{~F}_{p}\right)$ except the point of infinity $P_{\infty}=$ $(\infty, \infty)$. The computation of the square roots in the above formula was done by squaring the numbers $1,2, \ldots,(p-1) / 2$ and storing them for use for all points of the curve. The above method gives immediately the number of points on $E\left(F_{p}\right)$ :

$$
N_{p}=p-a_{p}+1
$$

and hence the value of $a_{p}$. This is obtained by $O(p)$ operations.

3.2. The Primary Decomposition of the Group. We obtain first the prime decomposition of $N_{p}$. The actual determination of the structure of the $l$-primary component is done as follows. If there are $l-1$ points of order $l$ in $E\left(F_{p}\right)$, then the $l$ primary component is cyclic. Otherwise the $l$-primary component has rank 2 , and the problem now becomes that of determining how the l-primary component breaks as a direct sum of two cyclic groups. Now if the rank of the l-primary part of $E\left(F_{p}\right)$ is 2 and $l^{2} \| N_{p}$ or $l^{3} \| N_{p}$, then

$$
E\left(\mathrm{~F}_{p}\right)=(Z / l Z) \oplus(Z / l Z) \quad \text { or } \quad{ }_{l} E\left(\mathbf{F}_{p}\right)=\left(Z / l^{2} Z\right) \oplus(Z / l Z),
$$

respectively. If the rank is 2 and $l^{m} \| N_{p}$ with $m \geqslant 4$, then let $m_{i}$ be the number of points of order $l^{i}$ for $i=1,2, \ldots,[m / 2]$. Let $j$ be the largest $i$ such that $m_{i}=l^{2 i}$ $l^{2 i-2}$, then the $l$-primary component of $E\left(\mathrm{~F}_{p}\right)$ is

$$
E\left(\mathrm{~F}_{p}\right)=\left(Z / l^{j} Z\right) \oplus\left(Z / l^{m-j} Z\right)
$$

Remarks. (1) An important fact used implicitly in the computation of $E\left(\mathbf{F}_{p}\right)$ is that the kernel ${ }_{m} E$ of multiplication by $m$ in the elliptic curve $E\left(\overline{\mathbf{F}}_{p}\right)$ is a product of two cyclic groups of order $m$ and carries a natural symplectic structure. Thus if all the $m^{2}$ points of ${ }_{m} E$ have coordinates in the field $\mathbf{F}_{p}$, then $\mathbf{F}_{p}$ contains all $m$ th root of unity, i.e. $m \mid(p-1)$. This gives immediately the fact that if $N_{p}$ and $p-1$ are relatively prime, then $E\left(\mathbf{F}_{p}\right)$ is cyclic, or equivalently if $a_{p}-2$ and $p-1$ are relatively prime, 
then $E\left(\mathbf{F}_{p}\right)$ is cyclic. This criterion greatly simplifies the computations since the size of $a_{p}-2$ is $O\left(p^{1 / 2}\right)$ by the "Riemann Hypothesis".

(2) We do not have to compute the order of all the points of $E\left(\mathbf{F}_{p}\right)$ in order to determine the group structure. If $l^{i} \| N_{p}$, we only need to know at most the number of points of order not larger than $l^{j},(j=[i / 2])$; this simply means that we have to iterate the group operation for all the points of $E\left(\mathbf{F}_{p}\right)$ at most $l^{j}-1,(j=[i / 2])$, times. The number of operations involved for every point is $O\left(l^{j}\right)=O\left(p^{1 / 2}\right)$, and since $N_{p}=$ $p-a_{p}+1=O(p)$, we get that the total number of operations involved in computing the group structure of $E\left(\mathrm{~F}_{p}\right)$ is at most $O\left(p^{3 / 2}\right)$.

(3) When two elliptic curves $E_{1}$ and $E_{2}$ are connected by an isogeny of degree $d$, the two groups $E_{1}\left(\mathrm{~F}_{p}\right)$ and $E_{2}\left(\mathrm{~F}_{p}\right)$ are the same except for the $l$-component for each prime $l \mid d$. As an example, we consider the case of the two elliptic curves

$$
E_{1}: y^{2}-y=x^{3}-x^{2} \text { and } E_{2}: y^{2}+y=x^{3}-x^{2}-10 x-20 .
$$

These are the curves $X_{1}(11)$ and $X_{0}(11)$, respectively, in the notation of Ogg [9] which in fact are connected by an isogeny of degree 5 . This leads to the following observations:

(i) The groups $E_{1}\left(\mathrm{~F}_{p}\right)$ and $E_{2}\left(\mathrm{~F}_{p}\right)$ are the same, except for the 5-component.

(ii) If $p \not \equiv \bmod (5)$, then the 5-component of both is cyclic and so the two groups are the same.

(iii) Using Kummer Theory, Ogg [9] shows that all 25 points on $E_{2}$ of order dividing 5 are rational over the cyclotomic field $Q\left(e^{2 \pi i / 5}\right)$, and for $p \equiv 1 \bmod (5)$ the group $E_{2}\left(\mathrm{~F}_{p}\right)$ is not cyclic.

In Table 2 we have given the structure of $E_{1}\left(F_{p}\right)$ from which we can easily compute the group $E_{2}\left(\mathbf{F}_{p}\right)$ using the remarks above.

Notation. In the subsequent tables the primary decomposition of a group will be written for simplicity in the form $\left(m_{1}, m_{2} m, \ldots, m_{k}\right)$ which stands for

$$
\left(Z / m_{1} Z\right) \oplus\left(Z / m_{2} Z\right) \oplus \cdots \oplus\left(Z / m_{k} Z\right) .
$$

4. Numerical Results.

4.1. Computations were carried out for six elliptic curves whose equations, $j$ invariants, conductors $N$ and discriminants $\Delta$ are given below. These curves were taken from Serre's article [10] where the Galois properties of their fields of $l$-division points are studied:

\section{Elliptic}

TABLE A

\begin{tabular}{cllll} 
curve & \multicolumn{1}{c}{ Equation } & \multicolumn{1}{c}{ j-invariant } & Conductor & Discriminant \\
$E_{1}$ & $y^{2}-y=x^{3}-x^{2}$ & $-2^{12} / 11$ & 11 & -11 \\
$E_{2}$ & $y^{2}+y=x^{3}-x^{2}-10 x-20$ & $-2^{12} \cdot 31^{3} / 11^{5}$ & 11 & $-11^{5}$ \\
$E_{3}$ & $y^{2}+x y=x^{3}+x^{2}-2 x-7$ & $-11^{2}$ & $11^{2}$ & $-11^{4}$ \\
$E_{4}$ & $y^{2}+y=x^{3}+x^{2}$ & $-2^{12} / 43$ & 43 & -43 \\
$E_{5}$ & $y^{2}+x y+y=x^{3}-x$ & $-5^{6} / 2^{2} \cdot 7$ & $2 \cdot 7$ & $-2^{2} \cdot 7$ \\
$E_{6}$ & $y^{2}+y=x^{3}-x$ & $2^{13} \cdot 3^{3} / 37$ & 37 & 37
\end{tabular}

The original computations were carried out for primes in the range from 2 to 
5000. Table 1 records the traces of the Frobenius endomorphism $a_{p}$ for the above curves for primes in the range 2 to 2000 . The results we obtained for $E_{1}$ were compared with those given by Shimura in [11] and are not included here. Curves $E_{1}$ and $E_{2}$ are isogenous and thus have the same $a_{p}$ (Vélu [12]).

Tables 2, 3, 4 and 5 give the prime $p$ and the primary decomposition (notation: PD) of $E\left(F_{p}\right)$; the order $N_{p}=$ Card $E\left(F_{p}\right)$ can easily be computed from this data. In Table 2 the results for $E_{1}$ are presented and from these we can easily compute $E_{2}\left(F_{p}\right)$ using the remarks at the end of Section 3. Each table goes up to 500 and presents some additional primes.

As is well known, the curve $E_{1}$ has a rational point of order 5 and hence $5 \mid N_{p}$ for all $p \neq 11$. Similarly $E_{5}$ has a rational point of order 6 and hence $6 \mid N_{p}$ except for $p=2$ and $p=7$.

The computations suggest that the reduction of the same curve for various primes $p$ may lead to the same order $N_{p}$ but to nonisomorphic groups. Below we give several examples:

\begin{tabular}{|c|c|c|c|c|c|c|c|}
\hline \multirow[b]{2}{*}{ Curve } & \multicolumn{7}{|c|}{ TABLE B } \\
\hline & Prime & $N_{p}$ & $E\left(\mathbf{F}_{p}\right)$ & Curve & Prime & $N_{p}$ & $E\left(\mathbf{F}_{p}\right)$ \\
\hline$E_{1}$ & 557 & 560 & $(16,5,7)$ & $E_{4}$ & 719 & 712 & $(2,4,89)$ \\
\hline$E_{1}$ & 599 & 560 & $(2,8,5,7)$ & $E_{4}$ & 727 & 712 & $(8,89)$ \\
\hline$E_{2}$ & 1021 & 1000 & $(2,4,5,25)$ & $E_{5}$ & 1579 & 1620 & $(2,2,3,27,5)$ \\
\hline$E_{2}$ & 1031 & 1000 & $(8,5,25)$ & $E_{5}$ & 1667 & 1620 & $(2,2,81,5)$ \\
\hline$E_{2}$ & 967 & 1000 & $(8,125)$ & $E_{6}$ & 1301 & 1372 & $(8,3,53)$ \\
\hline$E_{3}$ & 4091 & 4180 & $(4,5,11,19)$ & $E_{6}$ & 1307 & 1372 & $(2,4,3,53)$ \\
\hline$E_{3}$ & 4201 & 4180 & $(2,2,5,11,19)$ & & & & \\
\hline
\end{tabular}

Other examples may be found in the tables below.

An interesting observation that was made for the curve $E_{1}$ of conductor 11 and $E_{3}$ of conductor $11^{2}$ is that $3 \mid$ Card $E_{1}\left(\mathrm{~F}_{p}\right)$ if and only if $3 \mid \mathrm{Card} E_{3}\left(\mathrm{~F}_{p}\right)$. Also, the 3-primary component of $E_{1}\left(\mathrm{~F}_{p}\right)$ splits if and only if the 3-primary component of $E_{3}\left(F_{p}\right)$ splits. These observations can be checked in Tables 2 and 3. Below we give examples of the simultaneous splitting.

\begin{tabular}{|c|c|c|c|c|}
\hline$P$ & $N_{p}$ & $\begin{array}{l}\text { TABLE } \\
E_{1}\left(\mathbf{F}_{p}\right)\end{array}$ & $N_{p}$ & $E_{3}\left(\mathbf{F}_{p}\right)$ \\
\hline 337 & 360 & $(8,3,3,5)$ & 351 & $(3,9,13)$ \\
\hline 523 & 540 & $(4,3,9,5)$ & 540 & $(4,3,9,5)$ \\
\hline 1087 & 1080 & $(2,4,3,9,5)$ & 1134 & $(2,3,27,7)$ \\
\hline 2437 & 2520 & $(8,3,3,5,7)$ & 2520 & $(2,4,3,3,5,7)$ \\
\hline 2719 & 2790 & $(2,3,3,5,31)$ & 2664 & $(8,3,3,37)$ \\
\hline 2749 & 2700 & $(4,3,9,25)$ & 2673 & $(3,81,11)$ \\
\hline 3331 & 3375 & $(3,9,5,25)$ & 3312 & $(16,3,3,23)$ \\
\hline 3469 & 3555 & $(3,3,5,79)$ & 3429 & $(3,9,127)$ \\
\hline 3709 & 3690 & $(2,3,3,5,41)$ & 3753 & $(3,9,139)$ \\
\hline 4003 & 4050 & $(2,3,27,25)$ & 3960 & $(8,3,3,5,11)$ \\
\hline 4483 & 4590 & $(2,3,9,5,17)$ & 4500 & $(4,3,3,125)$ \\
\hline 4801 & 4725 & $(3,9,5,5,7)$ & 4779 & $(3,27,59)$ \\
\hline
\end{tabular}

The above are all the examples that appear in the range up to 4963 . 
TABLE 1. Traces of Frobenius




TABLE 1 (Continued)

\begin{tabular}{|c|c|c|c|c|c|c|c|c|c|c|c|}
\hline$P$ & $E_{1}, E_{2}$ & $E_{3}$ & $E_{4}$ & $E_{5}$ & $E_{6}$ & $p$ & $E_{1}, E_{2}$ & $E_{3}$ & $E_{4}$ & $\mathrm{E}_{5}$ & $\mathrm{E}_{6}$ \\
\hline $\begin{array}{l}859 \\
853\end{array}$ & & & & $\begin{array}{r}14 \\
-24\end{array}$ & & $\begin{array}{r}1433 \\
3439\end{array}$ & $\begin{array}{r}54 \\
0\end{array}$ & $\begin{array}{l}-9 \\
42\end{array}$ & $\begin{array}{l}-35 \\
-42\end{array}$ & $\begin{array}{l}42 \\
24\end{array}$ & -54 \\
\hline $\begin{array}{l}863 \\
877\end{array}$ & $\begin{array}{r}24 \\
-12\end{array}$ & $\begin{array}{l}-54 \\
-27\end{array}$ & $\begin{array}{l}-6 \\
41\end{array}$ & $\begin{array}{l}-24 \\
-22\end{array}$ & $\begin{array}{r}-24 \\
50\end{array}$ & $\begin{array}{l}1439 \\
1447\end{array}$ & $\begin{array}{r}0 \\
28\end{array}$ & $\begin{array}{r}42 \\
-38\end{array}$ & $\begin{array}{r}-42 \\
22\end{array}$ & $\begin{array}{r}24 \\
-64\end{array}$ & $\begin{array}{l}-53 \\
-57\end{array}$ \\
\hline 881 & $\begin{array}{l}-1 c \\
-43\end{array}$ & $\begin{array}{r}-21 \\
35\end{array}$ & 37 & -54 & $\begin{array}{r}30 \\
-14\end{array}$ & $\begin{array}{l}1441 \\
1451\end{array}$ & $\begin{array}{l}20 \\
52\end{array}$ & 22 & -50 & -48 & 20 \\
\hline 883 & 4 & -20 & 31 & 20 & 48 & 1453 & -71 & 13 & -10 & 50 & 47 \\
\hline 887 & $\begin{array}{l}-22 \\
-72\end{array}$ & -46 & -22 & -36 & 25 & 1459 & -20 & 64 & -23 & 38 & -37 \\
\hline 907 & $\begin{array}{r}-12 \\
12\end{array}$ & 12 & 47 & 44 & $\begin{array}{l}52 \\
26\end{array}$ & 1.71 & 22 & 28 & -1 & 32 & \\
\hline $\begin{array}{l}911 \\
919\end{array}$ & $\begin{array}{l}12 \\
10\end{array}$ & $\begin{array}{r}-24 \\
28\end{array}$ & $\begin{array}{l}-22 \\
-49\end{array}$ & 48 & $\begin{array}{r}26 \\
-58\end{array}$ & 1481 & 32 & -13 & 52 & $\begin{array}{r}-6 \\
-46\end{array}$ & -3 \\
\hline $\begin{array}{l}919 \\
929\end{array}$ & $\begin{array}{r}10 \\
-30\end{array}$ & $\begin{array}{r}28 \\
-21\end{array}$ & $\begin{array}{r}-49 \\
-6\end{array}$ & $\begin{array}{r}56 \\
6\end{array}$ & $\begin{array}{r}-58 \\
18\end{array}$ & $\begin{array}{l}1483 \\
1437\end{array}$ & $\begin{array}{l}49 \\
58\end{array}$ & $\begin{array}{l}64 \\
34\end{array}$ & $\begin{array}{l}19 \\
24\end{array}$ & $\begin{array}{r}-46 \\
0\end{array}$ & -3 \\
\hline 937 & & 23 & 32 & 2 & 37 & 489 & -15 & 30 & 40 & -58 & -2 \\
\hline 914 & $\begin{array}{r}42 \\
-27\end{array}$ & -27 & 33 & -24 & -10 & 1493 & -36 & -27 & 21 & 30 & 6 \\
\hline $\begin{array}{l}947 \\
953\end{array}$ & $\begin{array}{r}-27 \\
34\end{array}$ & $\begin{array}{r}-42 \\
31\end{array}$ & $\begin{array}{r}-33 \\
22\end{array}$ & $\begin{array}{r}24 \\
-54\end{array}$ & $\begin{array}{l}12 \\
61\end{array}$ & 1499 & 55 & $\begin{array}{r}-20 \\
52\end{array}$ & $\begin{array}{l}26 \\
41\end{array}$ & $\begin{array}{r}-12 \\
0\end{array}$ & -6 \\
\hline $\begin{array}{l}953 \\
967\end{array}$ & $\begin{array}{r}34 \\
-32\end{array}$ & $\begin{array}{l}31 \\
22\end{array}$ & $\begin{array}{l}22 \\
37\end{array}$ & $\begin{array}{r}-54 \\
32\end{array}$ & $\begin{array}{r}61 \\
-14\end{array}$ & $\begin{array}{l}1511 \\
1523\end{array}$ & $\begin{array}{r}37 \\
-41\end{array}$ & $\begin{array}{l}52 \\
34\end{array}$ & $\begin{array}{l}41 \\
60\end{array}$ & -72 & -3 \\
\hline 971 & 47 & 2 & -13 & -6 & -8 & 1531 & 32 & 56 & 0 & 2 & 1 \\
\hline $\begin{array}{l}977 \\
083\end{array}$ & $\begin{array}{r}-27 \\
39\end{array}$ & $\begin{array}{l}-57 \\
-36\end{array}$ & $\begin{array}{r}34 \\
-24\end{array}$ & -6 & 28 & 1543 & -36 & 24 & -56 & $\begin{array}{r}32 \\
-34\end{array}$ & $\begin{array}{r}-1 \\
-14\end{array}$ \\
\hline $\begin{array}{l}983 \\
991\end{array}$ & $\begin{array}{l}39 \\
-8\end{array}$ & $\begin{array}{l}-36 \\
-20\end{array}$ & $\begin{array}{r}-24 \\
2\end{array}$ & $\begin{array}{l}-36 \\
-16\end{array}$ & $\begin{array}{r}99 \\
-18\end{array}$ & $\begin{array}{l}1549 \\
1553\end{array}$ & $\begin{array}{l}-15 \\
-56\end{array}$ & $\begin{array}{r}99 \\
34\end{array}$ & $\begin{array}{l}14 \\
12\end{array}$ & $\begin{array}{r}-34 \\
42\end{array}$ & -1 \\
\hline 997 & 38 & 53 & 4 & 8 & -42 & 1559 & -60 & 6 & -44 & -12 & -4 \\
\hline 1009 & $\begin{array}{r}-10 \\
30\end{array}$ & $\begin{array}{l}-49 \\
-42\end{array}$ & $\begin{array}{l}-18 \\
-22\end{array}$ & $\begin{array}{l}-34 \\
-36\end{array}$ & $\begin{array}{r}-47 \\
36\end{array}$ & 1567 & $\begin{array}{r}-52 \\
\end{array}$ & -10 & 32 & -16 & \\
\hline $\begin{array}{l}1013 \\
1019\end{array}$ & $\begin{array}{r}39 \\
-10\end{array}$ & $\begin{array}{l}-42 \\
-46\end{array}$ & $\begin{array}{l}-22 \\
-30\end{array}$ & $\begin{array}{r}-36 \\
36\end{array}$ & $\begin{array}{l}36 \\
46\end{array}$ & & $\begin{array}{l}-28 \\
-30\end{array}$ & $\begin{array}{r}20 \\
6\end{array}$ & $\begin{array}{r}33 \\
-19\end{array}$ & $\begin{array}{r}66 \\
-40\end{array}$ & 3 \\
\hline $\begin{array}{l}1019 \\
1021\end{array}$ & 22 & -2 & -14 & -4 & -62 & $\begin{array}{l}1579 \\
1583\end{array}$ & $\begin{array}{r}-30 \\
34\end{array}$ & $\begin{array}{r}0 \\
-14\end{array}$ & $\begin{array}{r}-19 \\
9\end{array}$ & 0 & -4 \\
\hline 103 & $\begin{array}{r}32 \\
-16\end{array}$ & 50 & 46 & 0 & -4 & 1597 & -32 & 24 & -74 & -58 & \\
\hline 15 & $\begin{array}{r}-16 \\
5\end{array}$ & 11 & 13 & 26 & & 1601 & $\frac{2}{3 \pi}$ & -49. & 13 & 66 & -2 \\
\hline $\begin{array}{l}103 \\
104\end{array}$ & $\begin{array}{r}5 \\
-55\end{array}$ & $\begin{array}{l}-10 \\
-25\end{array}$ & $\begin{array}{r}34 \\
6\end{array}$ & $\begin{array}{r}-4 \\
-30\end{array}$ & $\begin{array}{r}-59 \\
-4\end{array}$ & 1607 & 33 & $\begin{array}{l}24 \\
35\end{array}$ & 37 & $\begin{array}{l}48 \\
26\end{array}$ & $\begin{array}{l}72 \\
-4\end{array}$ \\
\hline $\begin{array}{l}1049 \\
1051\end{array}$ & $\begin{array}{r}-55 \\
2\end{array}$ & $\begin{array}{l}-25 \\
-16\end{array}$ & $\begin{array}{r}0 \\
-60\end{array}$ & $\begin{array}{r}-30 \\
44\end{array}$ & $\begin{array}{l}-4 \\
-16\end{array}$ & $\begin{array}{l}1609 \\
1613\end{array}$ & $\begin{array}{r}-10 \\
-6\end{array}$ & $\begin{array}{r}35 \\
9\end{array}$ & $\begin{array}{r}14 \\
-80\end{array}$ & $\begin{array}{r}20 \\
-72\end{array}$ & - \\
\hline 1061 & -13 & -43 & -40 & -30 & -62 & 1619 & -20 & -32 & -36 & -60 & - \\
\hline 1063 & $\begin{array}{r}44 \\
-20\end{array}$ & 20 & $\begin{array}{l}12 \\
32\end{array}$ & -16 & $\begin{array}{r}7 \\
-30\end{array}$ & 1621 & 22 & -47 & 36 & 74 & \\
\hline $\begin{array}{l}1069 \\
1087\end{array}$ & $\begin{array}{r}-20 \\
8\end{array}$ & $-4 \frac{1}{6}$ & $\begin{array}{l}32 \\
20\end{array}$ & $\begin{array}{l}8 \\
8\end{array}$ & $\begin{array}{r}-30 \\
12\end{array}$ & 1627 & $\begin{array}{l}78 \\
33\end{array}$ & $\begin{array}{l}0 \\
9\end{array}$ & $\begin{array}{r}47 \\
-56\end{array}$ & $\begin{array}{r}-10 \\
0\end{array}$ & -42 \\
\hline 1091 & -58 & -10 & 40 & 30 & 30 & $\begin{array}{l}1037 \\
1657\end{array}$ & $\begin{array}{l}33 \\
-2\end{array}$ & -2 & 1 & -34 & - \\
\hline & $\begin{array}{l}-51 \\
-42\end{array}$ & 30 & -46 & -22 & $\begin{array}{r}-36 \\
36\end{array}$ & 1663 & 4 & 34 & $-2 \overline{6}$ & 8 & -2 \\
\hline & $\begin{array}{l}-42 \\
-51\end{array}$ & $\begin{array}{l}39 \\
24\end{array}$ & $\begin{array}{l}12 \\
54\end{array}$ & $\begin{array}{l}-6 \\
48\end{array}$ & $\begin{array}{l}36 \\
-8\end{array}$ & 1667 & 48 & 6 & 24 & 48 & $\begin{array}{r}-52 \\
54\end{array}$ \\
\hline $\begin{array}{l}1105 \\
1109\end{array}$ & $\begin{array}{l}-51 \\
-30\end{array}$ & 42 & $\begin{array}{r}54 \\
0\end{array}$ & 36 & -35 & $\begin{array}{l}1669 \\
1693\end{array}$ & 50 & $\begin{array}{l}17 \\
66\end{array}$ & $\begin{array}{l}-14 \\
-26\end{array}$ & $\begin{array}{l}32 \\
56\end{array}$ & $\begin{array}{r}54 \\
-62\end{array}$ \\
\hline 1117 & 48 & -27 & 4 & -34 & 33 & 1697 & $\begin{array}{l}-40 \\
-42\end{array}$ & -9 & -18 & -42 & \\
\hline & 24 & 24 & 60 & -46 & -22 & 1699 & 40 & 34 & 50 & -10 & 6 \\
\hline & $\begin{array}{r}50 \\
2\end{array}$ & -13 & -26 & 50 & 50 & 1709 & -45 & 30 & -50 & 54 & \\
\hline $\begin{array}{l}1151 \\
1153\end{array}$ & $-31^{2}$ & $\begin{array}{l}-46 \\
-46\end{array}$ & $\begin{array}{r}-30 \\
21\end{array}$ & $\begin{array}{r}-12 \\
2\end{array}$ & $\begin{array}{r}-25 \\
18\end{array}$ & 1721 & $-\overline{3}$ & 78 & -45 & -6 & -2 \\
\hline $\begin{array}{l}1153 \\
1163\end{array}$ & $\begin{array}{r}-31 \\
34\end{array}$ & $\begin{array}{l}-46 \\
-38\end{array}$ & $\begin{array}{l}21 \\
32\end{array}$ & -60 & $\begin{array}{r}10 \\
-36\end{array}$ & $\begin{array}{l}1723 \\
1733\end{array}$ & $\begin{array}{r}-46 \\
-6\end{array}$ & $\begin{array}{r}-46 \\
6\end{array}$ & $\begin{array}{l}58 \\
45\end{array}$ & $\begin{array}{l}-4 \\
-6\end{array}$ & - \\
\hline 1171 & -3 & 12 & -52 & 20 & -22 & 1741 & 17 & -31 & -59 & -76 & 6 \\
\hline 118 & $\begin{array}{l}-18 \\
-12\end{array}$ & -3 & $\begin{array}{l}-40 \\
-48\end{array}$ & 60 & $\begin{array}{r}57 \\
-33\end{array}$ & 1747 & -57 & -24 & -52 & 15 & 2 \\
\hline & $\begin{array}{l}-12 \\
-21\end{array}$ & $\begin{array}{r}66 \\
-21\end{array}$ & $\begin{array}{r}-48 \\
32\end{array}$ & $\begin{array}{r}-12 \\
66\end{array}$ & $\begin{array}{l}-33 \\
-11\end{array}$ & 1753 & -4 & 19 & 4 & -46 & \\
\hline $\begin{array}{l}1193 \\
1201 \\
1\end{array}$ & $\begin{array}{r}-21 \\
2\end{array}$ & $\begin{array}{r}-21 \\
23\end{array}$ & $\begin{array}{r}22 \\
-55\end{array}$ & $\begin{array}{l}00 \\
14\end{array}$ & 44 & $\begin{array}{l}1759 \\
1777\end{array}$ & $\begin{array}{r}-40 \\
8\end{array}$ & $\begin{array}{l}-22 \\
-82\end{array}$ & $\begin{array}{r}28 \\
-62\end{array}$ & $\begin{array}{l}32 \\
50\end{array}$ & $\begin{array}{l}c \\
-6\end{array}$ \\
\hline $\begin{array}{l}1213 \\
1217\end{array}$ & $\begin{array}{l}-41 \\
-42\end{array}$ & $\begin{array}{r}46 \\
-57\end{array}$ & $\begin{array}{l}-59 \\
-38\end{array}$ & $\begin{array}{l}26 \\
-6\end{array}$ & $\begin{array}{l}-12 \\
-41\end{array}$ & 1783 & $\begin{array}{r}59 \\
\end{array}$ & $\begin{array}{r}-64 \\
1 ?\end{array}$ & 34 & -16 & \\
\hline $\begin{array}{l}1221 \\
1229\end{array}$ & 14 & 56 & -6 & -24 & 30 & $\begin{array}{l}1787 \\
1789\end{array}$ & $\begin{array}{r}-57 \\
10\end{array}$ & $\begin{array}{r}12 \\
-35\end{array}$ & $\begin{array}{r}-65 \\
78\end{array}$ & $\begin{array}{r}72 \\
-10\end{array}$ & \\
\hline $\begin{array}{l}1229 \\
1231\end{array}$ & 60 & 6 & 3 & 30 & -48 & $\begin{array}{l}1,09 \\
1801\end{array}$ & $\begin{array}{l}10 \\
52\end{array}$ & $\begin{array}{l}-73 \\
-38\end{array}$ & 73 & -34 & -16 \\
\hline $\begin{array}{l}1231 \\
1237\end{array}$ & -18 & 0 & -26 & -28 & -19 & 1811 & 12 & 42 & -26 & 42 & \\
\hline $\begin{array}{l}123 \\
12 I_{1}\end{array}$ & 18 & 45 & -20 & -40 & -42 & 1823 & -56 & 28 & -3 & -24 & \\
\hline $12 \%$ & $1: 0$ & -5 & -50 & 26 & -13 & 18 & -43 & -76 & -8 & -40 & \\
\hline 125 & -25 & 56 & -12 & 18 & & 1847 & -5 & -22 & 29 & 12 & \\
\hline $\begin{array}{l}127 \\
127\end{array}$ & $\begin{array}{l}-47 \\
-15\end{array}$ & 46 & $\begin{array}{r}-20 \\
-20\end{array}$ & 24 & $\begin{array}{r}-24 \\
64\end{array}$ & 1861 & 62 & -43 & -16 & 8 & \\
\hline 127 & $\begin{array}{l}-15 \\
-36\end{array}$ & $\begin{array}{l}211 \\
412\end{array}$ & $\begin{array}{r}20 \\
37\end{array}$ & 20 & 36 & 18 & 28 & -38 & 14 & 50 & \\
\hline & $\begin{array}{r}-30 \\
0\end{array}$ & $\begin{array}{r}42 \\
-21\end{array}$ & $\begin{array}{r}-37 \\
2\end{array}$ & $\begin{array}{r}0 \\
30\end{array}$ & $\begin{array}{r}-36 \\
48\end{array}$ & 1871 & -3 & -4 & 72 & 48 & \\
\hline $\begin{array}{l}125 \\
129\end{array}$ & -8 & $\begin{array}{l}-21 \\
-111\end{array}$ & $\begin{array}{r}2 \\
-9\end{array}$ & $\begin{array}{r}30 \\
-58\end{array}$ & 40 & $\begin{array}{l}1873 \\
1877\end{array}$ & $\begin{array}{l}-6 \\
18\end{array}$ & $\begin{array}{r}-42 \\
42\end{array}$ & $\begin{array}{r}-55 \\
16\end{array}$ & $\begin{array}{r}2 \\
78\end{array}$ & \\
\hline 129 & 48 & -33 & -38 & -34 & -38 & 1879 & -35 & -2 & 54 & 44 & -7 \\
\hline $1 \geq 0$ & $\begin{array}{l}27 \\
30\end{array}$ & $\begin{array}{l}57 \\
27\end{array}$ & $\begin{array}{l}39 \\
39\end{array}$ & -48 & $\begin{array}{l}30 \\
56\end{array}$ & 1889 & 70 & -5 & -45 & 18 & \\
\hline 130 & $\begin{array}{l}39 \\
28\end{array}$ & -2 & $\begin{array}{r}39 \\
-27\end{array}$ & $\begin{array}{r}8 \\
42\end{array}$ & $\begin{array}{l}56 \\
36\end{array}$ & 1901 & 77 & $\begin{array}{l}-79 \\
-58\end{array}$ & $\begin{array}{r}3 \\
-52\end{array}$ & -18 & -6 \\
\hline $\begin{array}{l}130 \\
1319\end{array}$ & -30 & -66 & -18 & $\begin{array}{r}-24 \\
\end{array}$ & -4 & $\begin{array}{l}1907 \\
1913\end{array}$ & $\begin{array}{l}-52 \\
-36\end{array}$ & $\begin{array}{l}-58 \\
-33\end{array}$ & $\begin{array}{l}-53 \\
-54\end{array}$ & $\begin{array}{l}42 \\
54\end{array}$ & \\
\hline 1321 & 117 & & 66 & 62 & 35 & 1931 & -18 & 72 & -50 & -30 & \\
\hline $\begin{array}{l}132 \\
136\end{array}$ & $\begin{array}{l}68 \\
12\end{array}$ & $\begin{array}{r}-46 \\
6\end{array}$ & $\begin{array}{r}66 \\
0\end{array}$ & $\begin{array}{r}32 \\
-18\end{array}$ & $6 \overline{6}$ & 1933 & 54 & 6 & 31 & 62 & - \\
\hline $\begin{array}{l}1361 \\
13667\end{array}$ & $\begin{array}{r}12 \\
-72\end{array}$ & -42 & $\begin{array}{r}0 \\
-12\end{array}$ & $\begin{array}{l}-10 \\
-24\end{array}$ & 62 & & -40 & -10 & 33 & 84 & $\begin{array}{l}01 \\
-4\end{array}$ \\
\hline 1373 & 39 & 9 & -45 & -30 & -2 & $\begin{array}{l}1951 \\
1973\end{array}$ & $\begin{array}{r}-23 \\
79\end{array}$ & $\begin{array}{l}-14 \\
-47\end{array}$ & $\begin{array}{l}-17 \\
-81\end{array}$ & $\begin{array}{l}-04 \\
-60\end{array}$ & - \\
\hline 1381 & -68 & 61 & 52 & -22 & 63 & 1979 & 30 & 0 & 44 & 30 & \\
\hline 139 & 60 & -54 & 32 & -40 & -25 & 1987 & -22 & 86 & 56 & -82 & -6 \\
\hline $\begin{array}{l}1409 \\
1423\end{array}$ & $\begin{array}{r}-15 \\
29\end{array}$ & $\begin{array}{r}-9 \\
-58\end{array}$ & $\begin{array}{l}42 \\
29\end{array}$ & $\begin{array}{l}18 \\
56\end{array}$ & $\begin{array}{r}-1 \\
-34\end{array}$ & 1993 & -66 & -54 & 45 & $\begin{array}{l}14 \\
40\end{array}$ & \\
\hline $\begin{array}{l}1423 \\
1427\end{array}$ & $\begin{array}{r}29 \\
-12\end{array}$ & -EO & $\begin{array}{r}29 \\
-12\end{array}$ & $\begin{array}{r}50 \\
-30\end{array}$ & $\begin{array}{l}-34 \\
-31\end{array}$ & $\begin{array}{l}1997 \\
1999\end{array}$ & $\begin{array}{l}-72 \\
-20\end{array}$ & $\begin{array}{l}-27 \\
-38\end{array}$ & $\begin{array}{l}22 \\
63\end{array}$ & $\begin{array}{l}-78 \\
-16\end{array}$ & \\
\hline & & & & & & & & & & & \\
\hline
\end{tabular}


TABLE 2. $E_{1}: y^{2}-y=x^{3}-x^{2}$

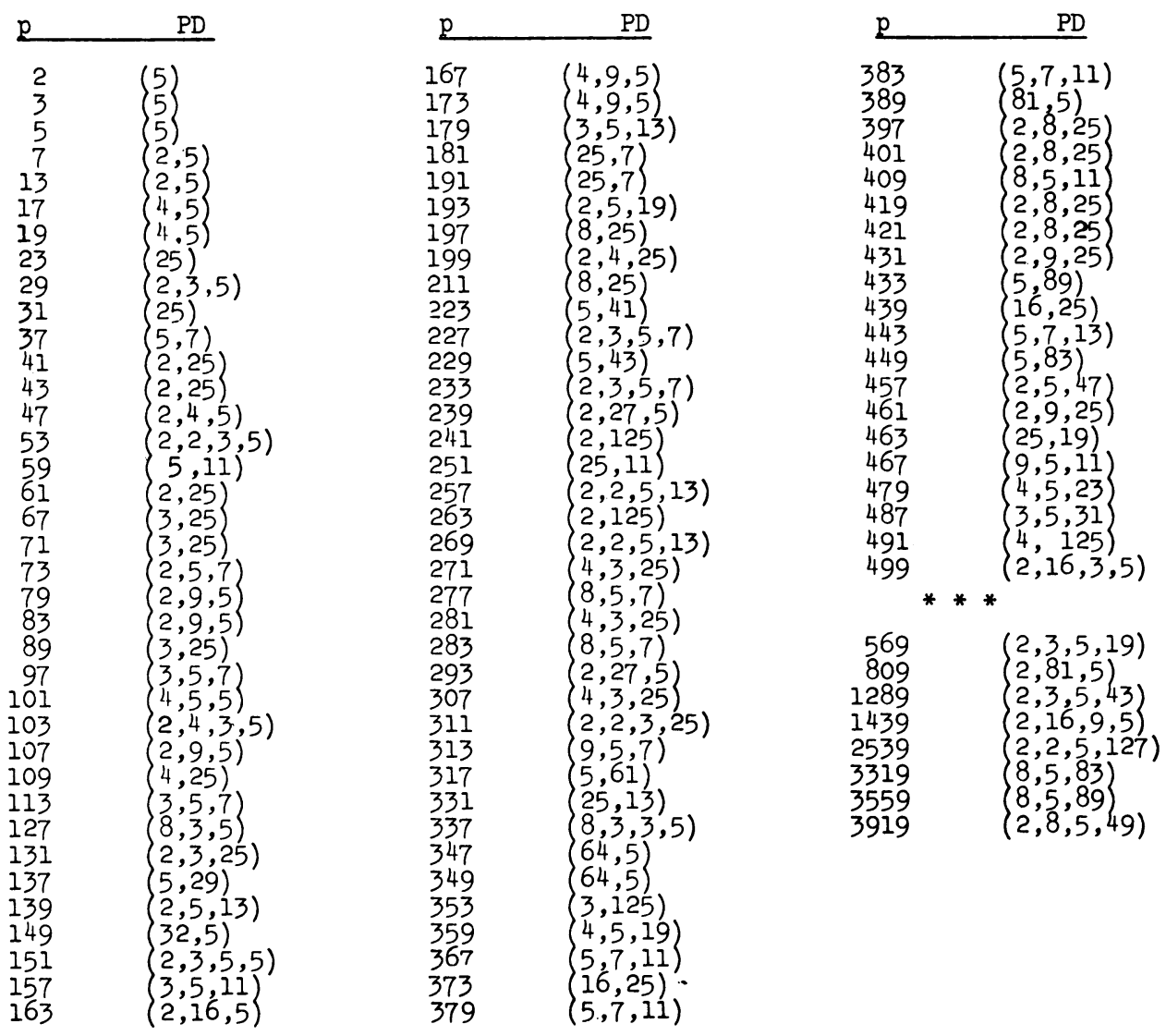

4.3. Densities. For a fixed elliptic curve $E$ defined over the rationals and a fixed prime $l$, a natural question to ask is, what is the set of primes $p$ such that $l$ divides Card $E\left(\mathrm{~F}_{p}\right)$. We will denote this set by $P_{l}(E)$. We also denote by $\operatorname{Sp}_{l}(E)$ the set of primes $p$ such that $l$-primary part splits. If $E$ has complex multiplications, then the splitting field of the $l$-division equation is abelian over the corresponding imaginary quadratic field; and hence $P_{l}(E)$ can be characterized by congruences. The elliptic curves investigated here have no complex multiplication, and thus the splitting field of the $l$-division equation is not solvable in general, and $P_{l}(E)$ cannot be characterized by congruences; however, the Čebotarev Density Theorem can be applied in this situation to obtain that $P_{l}(E)$ has density. The actual theoretical computation of the density of $P_{l}(E)$ is done by using Serre's results concerning the $l$-division fields associated with $E$. In the case $l=2$, we obtain that the Dirichlet Density of $P_{2}\left(E_{1}\right)$ is $2 / 3$. Furthermore we also get that the density of primes for which the 2-primary component of $E_{1}\left(F_{p}\right)$ splits $1 / 6$ (see Heilbronn, p. 228 or Tate-Serre, p. 354 in [6]). A more detailed investigation will appear elsewhere.

The frequences of primes $p$ less than 5000 for which

$$
p \in P_{l}(E) \text { or } p \in \mathrm{Sp}_{l}(E)
$$

for the curves studied here are given in the following table. 
TABLE D

(Relative Frequencies)

$\begin{array}{ccccccccc}\text { Curve } & 2 \mid N_{p} & \begin{array}{c}\text { 2-component } \\ \text { splits }\end{array} & 3 \mid N_{p} & \begin{array}{c}\text { 3-component } \\ \text { splits }\end{array} & 5 \mid N_{p} & 7 \mid N_{p} & \begin{array}{c}E\left(\mathbf{F}_{p}\right) \\ \text { cyclic }\end{array} & N_{p} \text { prime } \\ E_{1} & 0.67 & 0.16 & 0.44 & 0.018 & 1.0 & 0.17 & 0.62 & 0 \\ E_{2} & 0.67 & 0.16 & 0.44 & 0.018 & 1.0 & 0.17 & 0.62 & 0 \\ E_{3} & 0.66 & 0.16 & 0.44 & 0.016 & 0.24 & 0.15 & 0.82 & 0.063 \\ E_{4} & 0.66 & 0.15 & 0.42 & 0.010 & 0.22 & 0.12 & 0.82 & 0.07 \\ E_{5} & 1.00 & 0.49 & 1.00 & 0.156 & 0.23 & 0.16 & 0.43 & 0 \\ E_{6} & 0.67 & 0.16 & 0.45 & 0.017 & 0.24 & 0.14 & 0.83 & 0.084\end{array}$

The last two columns correspond to the frequencies of primes for which $E\left(F_{p}\right)$ is cyclic.

4.4. Theorems and Conjectures. The above discussion of the numerical results and the tables suggest a few conjectures, some of which we could prove and others which are still open. In Table $C$ we gave some examples of prime pairs $(p, q)$ such that Card $E\left(\mathbf{F}_{p}\right)$ $=$ card $E\left(F_{p}\right)$. In the computations we found many other occurrences of such pairs which suggest that the number of these pairs is infinite. We also found many triplets.

TABLE 3. $y^{2}+x y=x^{3}+x^{2}-2 x-7$

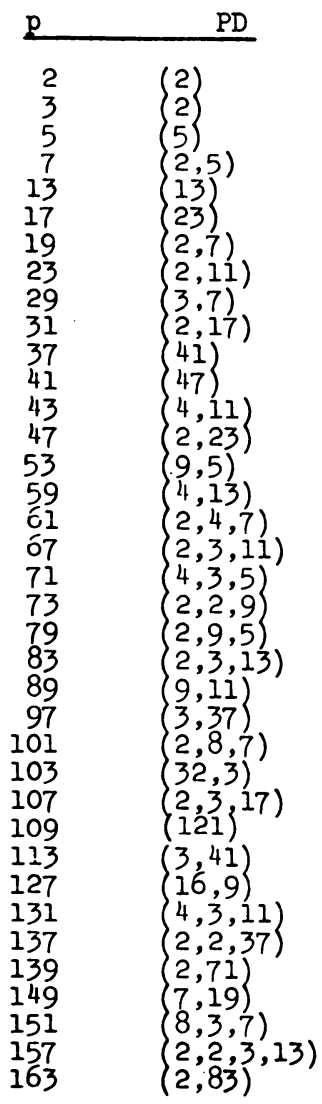

\begin{tabular}{|c|c|}
\hline$p$ & $P D$ \\
\hline $\begin{array}{l}167 \\
173 \\
179 \\
181 \\
191 \\
193 \\
197 \\
199 \\
211 \\
223 \\
227 \\
229 \\
233 \\
239 \\
241 \\
251 \\
257 \\
263 \\
269 \\
271 \\
277 \\
281 \\
283 \\
293 \\
307 \\
311 \\
313 \\
317 \\
331 \\
337 \\
347 \\
349 \\
353 \\
359 \\
367 \\
373 \\
379\end{array}$ & 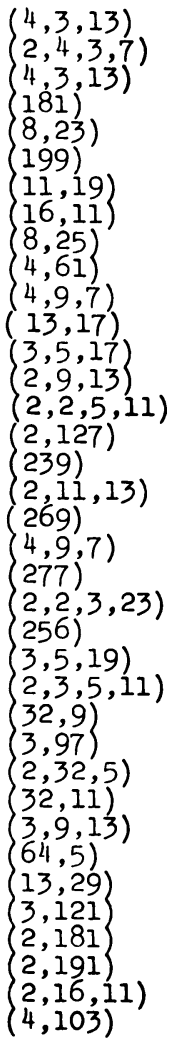 \\
\hline
\end{tabular}

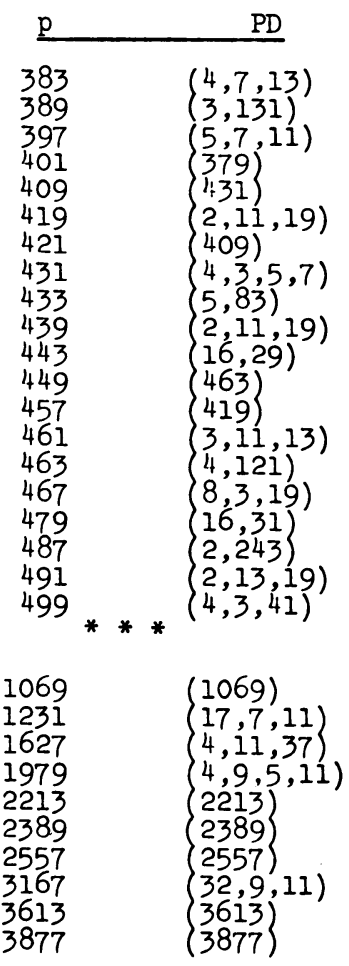


TABLE 4. $E_{4}: y^{2}+y=x^{3}+x^{2}$

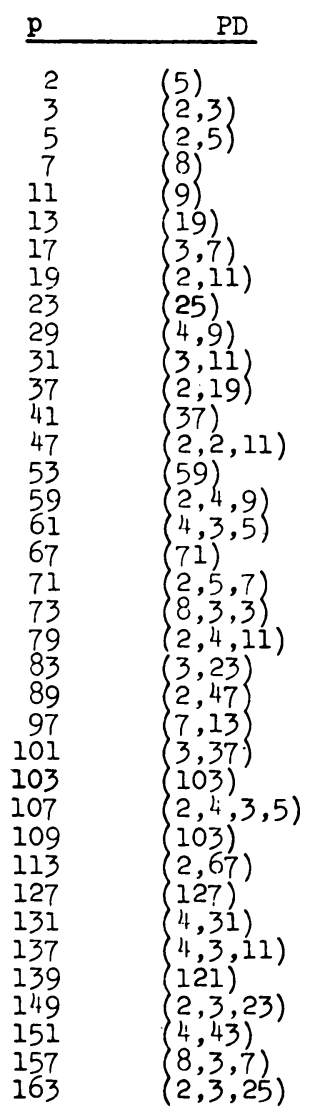

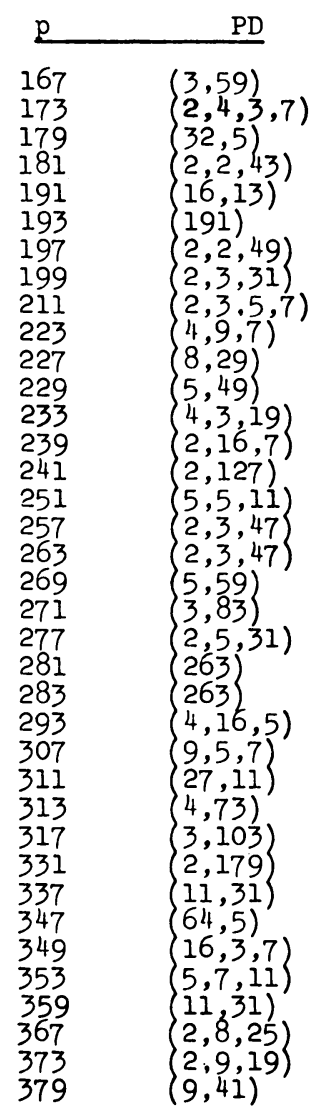
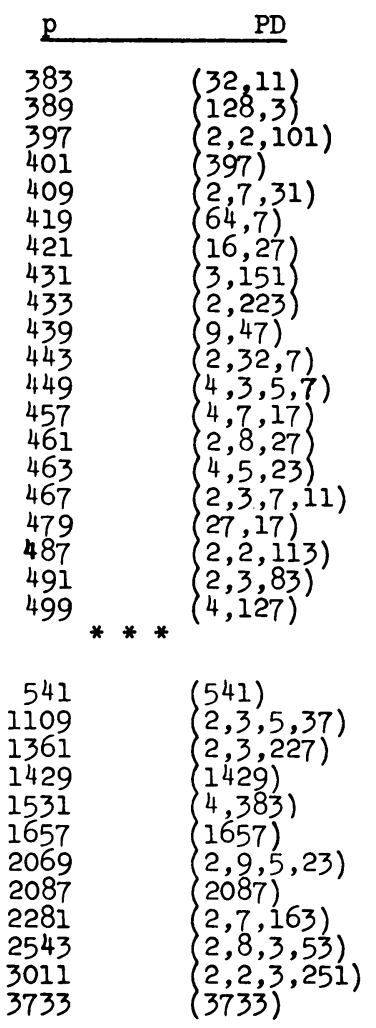

Given an elliptic curve $E$, it might be of interest to know the density of rational integers $n$ such that $n=$ card $E\left(F_{p}\right)$. A related question is that of the density of integers $n$ which can be traces of Frobenius for a given elliptic curve. The last column of Table D shows that except for trivial reasons $E\left(\mathrm{~F}_{p}\right)$ is a cyclic group of prime order for a substantial number of primes $p$. Nevertheless, this frequency seems to tend to zero. For an interesting discussion of a related problem, see Mazur [8].

Another question that arises is that of characterizing which finite abelian groups can be realized as $E\left(\mathrm{~F}_{p}\right)$ for some $E$ and some $p$. Clearly not all finite abelian groups can be so realized, and it would be of interest to know if the obvious necessary condition that each primary part should have rank at most two is also sufficient.

The computations suggest that the number of distinct prime divisors of card $E\left(\mathbf{F}_{p}\right)$ may be large. In this situation we can prove the following:

THEOREM. We have

$$
\lim _{p} \operatorname{Sup} \omega\left(N_{p}\right)=\infty
$$

where $\omega(n)=$ number of distinct prime divisors of $n$.

The proof of this theorem is given in [2]. 
TABLE 5. $E_{5}: y^{2}+x y+y=x^{3}-x$

$\underline{\underline{p}}$

\begin{tabular}{|c|}
\hline 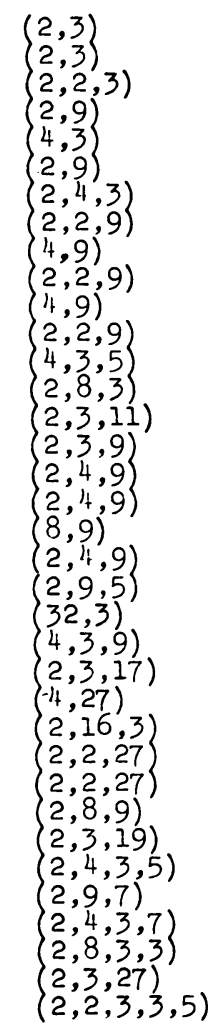 \\
\hline
\end{tabular}

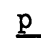

167
173
179
181
191
193
197
199
211
223
227
229
233
239
241
251
257
263
269
271
277
281
283
293
307
311
313
317
331
337
347
349
353
359
367
373
379
14
PD

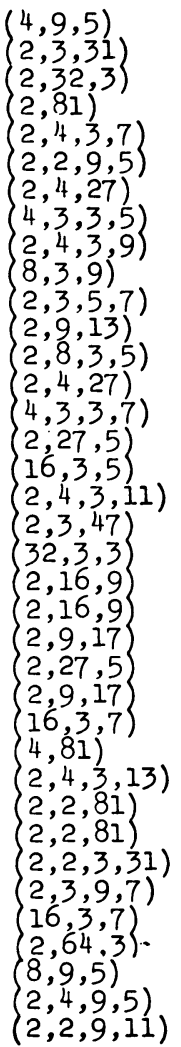

PD

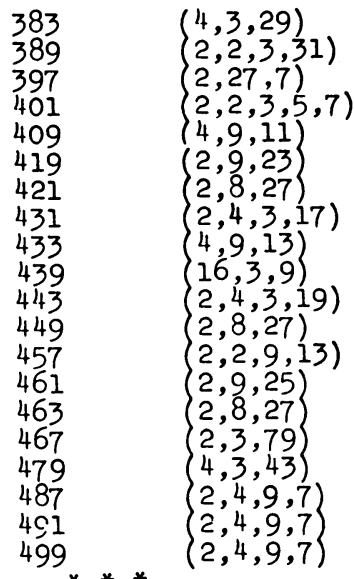

$\left\{\begin{array}{l}8,9,7) \\ 2,4,3,43) \\ 2,2,3,107\end{array}\right.$

$(16,3,31)$

$8,27,7)$

$2,8,9,11)$

$2,9,7,13)$

$2,4,3,5,17)$

$2,4,9,29$

$2,8,3,53)$

$2,2,9,79$

$(16,27,7)$

The discussion in the preceding paragraphs about the densities leads to the following result. Put:

$$
\begin{aligned}
& \pi(x)=\# \text { of primes less than } x \\
& D_{l}(x)=\#\left\{p \leqslant x: p \text { prime, } l \mid \text { card } E\left(F_{p}\right)\right\} \\
& \widetilde{D}_{l}(x)=\#\left\{p \leqslant x: p \in \operatorname{Sp}_{l}(E)\right\}, \\
& \hat{D}_{l}(x)=\#\left\{p \leqslant x: p \equiv-1 \bmod l \text { and } a_{p} \equiv 0 \bmod (l)\right\} .
\end{aligned}
$$

THEOREM. We have for almost all primes $l$

$$
\lim _{x \rightarrow \infty} D_{l}(X) / \pi(x)=C_{l}, \quad \lim _{x \rightarrow \infty} \widetilde{D}_{l}(x) / \pi(x)=\widetilde{C}_{l}, \quad \lim _{x \rightarrow \infty} \hat{D}_{l}(x) / \pi(x)=\hat{C}_{l},
$$

where

$$
C_{l}>\widetilde{C}_{l}=2 /(l-1)^{2} l(l+1) \text { and } \hat{C}_{l}>0
$$

The proof of this theorem will appear elsewhere.

For an elliptic curve $E, E\left(\mathbf{F}_{p}\right)$ is cyclic if each of its primary parts is of rank one. Therefore, we conjecture that the set of primes for which $E\left(F_{p}\right)$ is cyclic has a density given by $C(E)=\Pi_{l}^{*}\left(1-\widetilde{C}_{l}\right)$, where ${ }^{*}$ means that some correction should be made for the exceptional primes in the preceding theorem which is also the same as the set of exceptional primes in Serre's Theorem. Clearly $C(E)$ could be zero for trivial reasons 
TABLE 6. $E_{6}: y^{2}+y=x^{3}-x$

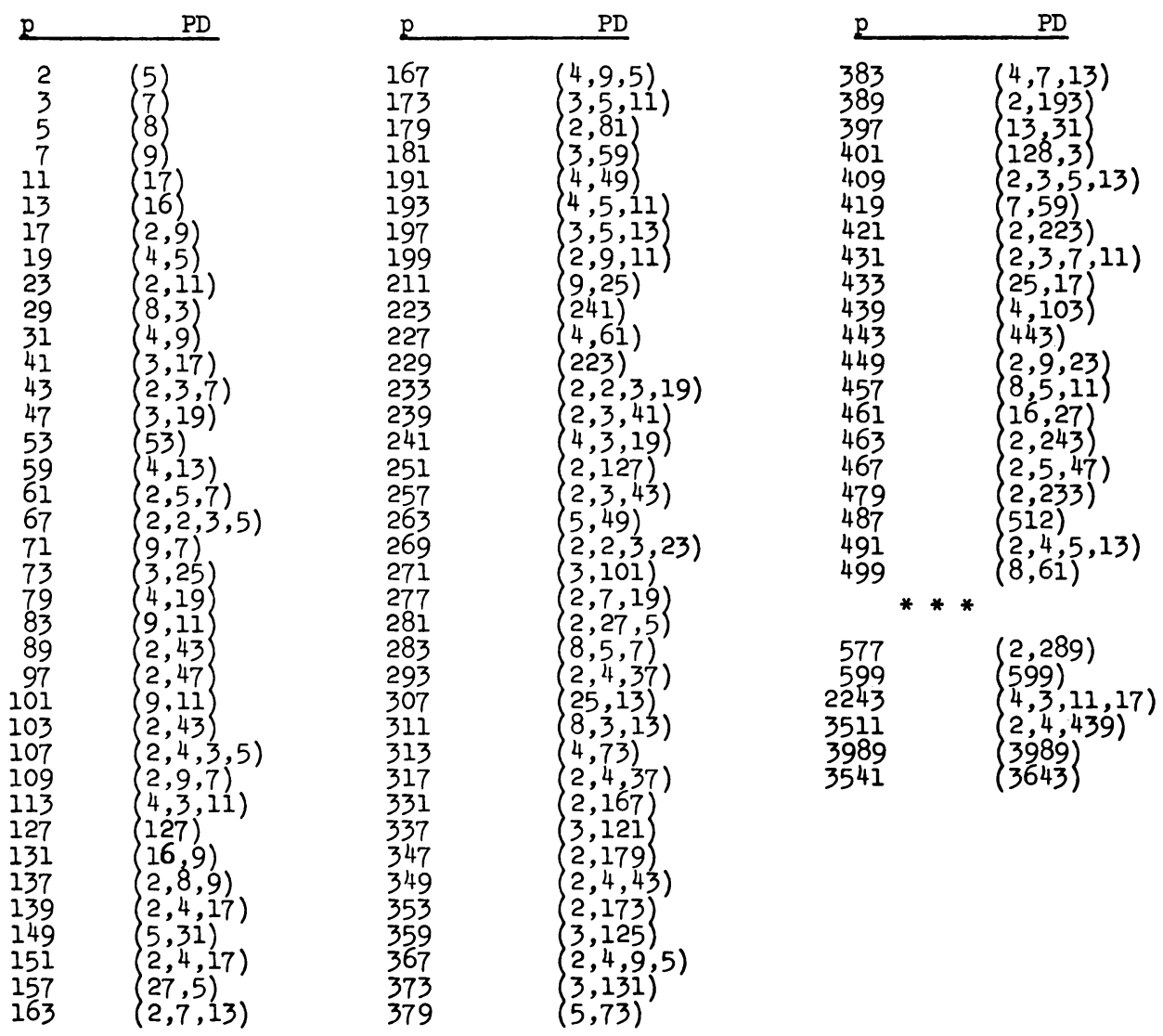

as happens, for example, for some of the modular curves, but the conjecture still makes sense once we divide $E$ by a suitable subgroup; for example, we can ask for the density of primes $p$ such that the group $\left(E_{1} / H\right)\left(F_{p}\right)$ is cyclic, where $H$ is the subgroup of order 5 generated by $(0,0)$.

Department of Mathematics

Texas A \& M University

College Station, Texas 77843

Department of Mathematics

University of Illinois at Urbana-Champaign

Urbana, Illinois 61801

1. B. J. BIRCH, Elliptic Curves and Modular Functions, Symposia Mathematica, vol. IV, (INDAM, Rome, 1968/69), Academic Press, London, 1970. MR 42 \#459.

2. I. BOROSH, C. MORENO \& H. PORTA, "Elliptic curves over finite fields. I," Proceedings of the 1972 Number Theory Conference, Boulder, Colorado.

3. I. BOROSH, C. MORENO \& H. PORTA, "Elliptic curves over finite fields. III." (In preparation.)

4. J. BRILLHART \& I. GERST, “On the prime divisors of polynomials," Amer. Math. Monthly, v. 78, 1971 , pp. 250-266. MR 43 \#4797.

5. J. W. S. CASSELS, "Diophantine equations with special reference to elliptic curves," J. London Math. Soc., v. 41, 1966, pp. 193-291; Corrigenda, ibid., v. 42, 1967, p. 183. MR 33 \#7299; 34 \#2523. 
6. J. W. S. CASSELS \& A. FRÖHLICH (Editors), Algebraic Number Theory, Academic Press, London; Thompson Book, Washington, D. C., 1967. MR 35 \#6500.

7. K. DOI \& H. NAGANUMA, "On the algebraic curves unirurmizea by arithmetical automorphic functions," Ann. of Math. (2), v. 86, 1967, pp. 449-460. MR 36 \#2618.

266.

8. B. MAZUR, "Rational points of abelian varieties," Invent. Math., v. 18, 1972, pp. 183-

9. A. P. OGG, "Rational points on certain elliptic modular curves," Number Theory Symposium, St. Louis, 1972.

10. J.-P. SERRE, "Propriétés Galoisiennes des points d'ordre fini des courbes elliptiques," Invent. Math., v. 15, 1972, pp. 259-331.

11. G. SHIMURA, "A reciprocity law in non-solvable extensions," J. Reine Angew. Math., v. 221, 1966, pp. 209-220. MR $32 \# 5637$.

12. J. VELU, "Courbes elliptiques sur $Q$ ayant bonne réduction en dehors de $\{11\}$," $C$. $R$. Acad. Sci. Paris Sér. A-B, v. 273, 1971, pp. A73-A75. MR 47 \#5004.

13. B. F. WYMAN, "What is a reciprocity law?," Amer. Math. Monthly, v. 79, 1972, pp. 571586. MR 46 \#7199. 